\title{
Health Equity Research in Nursing and Midwifery: Time to Expand Our Work
}

\author{
Deonni Stolldorf, PhD, RN; Hayley D. Germack, PhD, MHS, RN; Jordan Harrison, PhD, RN; Kathryn Riman, BSN, RN; \\ Heather Brom, PhD, RN; Michael Cary, PhD, RN; Heather Gilmartin, PhD, RN; Tammie Jones, MS, FACHE, CMPE; \\ Allison Norful, PhD, RN, ANP-BC; and Allison Squires, PhD, RN, FAAN
}

Keywords: Health disparities, health equity, midwifery, nursing, social determinants of health

$\mathrm{T}$ he World Health Organization (n.d.) named 2020 the "Year of the Nurse and Midwife" to both celebrate the contributions of nurses around the globe, recognize the challenges they face, and invest in and increase the nursing workforce. Indeed, the first half of the year has brought profound changes and challenges to nursing and midwifery. The COVID-19 pandemic has laid bare the structural inequities inherent in the US healthcare system. The collective "enough!” expressed by the public with regard to racism and discrimination toward Black and minority communities further raises the motivation to move our research toward addressing social justice issues. For nursing and midwifery, the time has come to address health equity in all its forms. The Interdisciplinary Research Group on Nursing Issues (IRGNI) of AcademyHealth has agreed to target its efforts in the coming years to address health equity. The IRGNI looks forward to using its platform to help share the unique perspectives of nurses and midwives in this important field of research.

Health equity is achieved by eliminating health disparities and inequalities (Braveman, 2006). Many of our fellow researchers use the terms "minority health," "disparities," "inequities," and "inequalities" interchangeably, yet they are conceptually distinct. With that, the IRGNI has reviewed and will adopt the revised definitions of minority health and health disparities published by the National Institute for Minority Health and Health Disparities (NIMHD). Alvidrez et al. (2019) published the revised definitions in a special issue of the American Journal of Public Health. The revised definitions read:

NIMHD defines minority health as all aspects of health and disease in one or more racial/ethnic minority populations as defined by the Office of Management and Budget, including Blacks/African Americans, Hispanics/Latinos, Asians, American Indians/Alaska Natives, and Native Hawaiians/other Pacific Islanders.
NIMHD defines a health disparity as a health difference, on the basis of one or more health outcomes, that adversely affects disadvantaged populations. According to the legislation that created NIMHD, a health disparity population is characterized by a pattern of poorer health outcomes, indicated by the overall rate of disease incidence, prevalence, morbidity, mortality, or survival in the population as compared with the general population. Current NIMHDdesignated health disparity populations include Office of Management and Budget-defined racial/ethnic minorities, socioeconomically disadvantaged populations, underserved rural populations, and sexual and gender minorities (which include lesbian, gay, bisexual, transgender, and gender-nonbinary or gender-nonconforming individuals). (p. S16)

Also important is the concept of healthcare disparities with issues of access and implementation of services that often disproportionately affect minority populations. Achieving healthcare equity means equitable access and patient experiences for all. The social determinants of health have also received increasing attention as important factors that can affect anyone at the individual, familial, community, and system levels (Carey \& Crammond, 2015). During the Year of the Nurse and Midwife, the IRGNI Advisory Committee is looking forward to open discussions regarding health equity and the revised NIMHD definitions.

We are proud to present this year's collection of IRGNI research abstracts. This work highlights how nurses and midwives can systematically move toward addressing health inequities, health inequalities, health disparities, and the social determinants of health. These abstracts cross the lifespan and focus on communities of color, understudied populations, and the places where we seek healthcare services. We hope readers will find new insights into the health of populations and their health-seeking behaviors. These findings will inform new directions for research, policy, and regulation and will strengthen the evidence base to dismantle sources of structural discrimination in the U.S. healthcare system. 


\section{Missed Nursing Care in Alabama: Frequency and Associated Factors}

Authors: Caitlin M. Campbell, BSN, RN; Aoyjai Prapanjaroensin, PhD, BSN; Colleen V. Anusiewicz, BSN, RN; Marianne Baernholdt, PhD, MPH, RN, FAAN; Terry Jones, PhD, RN; Patricia A. Patrician, PhD, RN, FAAN

Objective: Missed nursing care (MNC) threatens the quality and safety of care patients receive from nurses. Internationally, $55 \%$ to 98\% of nurses report not completing at least one basic nursing care activity while working their shifts. Nurses are fundamental members of the healthcare team and are crucial to ensuring the delivery of high quality, safe patient care. Nursing care in Alabama has been understudied despite it being a state with many known health concerns. Exploring areas for quality improvement in healthcare delivery, and specifically in nursing care, in Alabama is warranted. The objective of this study was to determine the frequency of MNC in Alabama hospitals, identify the organizational and nursing factors associated with $\mathrm{MNC}$, and predict overall MNC by associated factors.

Study Design: A secondary data analysis was conducted using cross-sectional data from the Alabama Hospital Staff Nurse Study, which is a study of inpatient staff nurses in Alabama. Data collection occurred between July 2018 and January 2019. MNC was measured using the Perceived Implicit Rationing of Nursing Care Instrument. To determine the organizational factors associated with MNC, the Practice Environment Scale of the Nursing Work Index was used to measure the nursing work environment. Additionally, single-item measures were used to examine nurse job satisfaction, unit type, and nurse-reported quality of care. Descriptive statistics were obtained to determine the frequency of MNC activities. Bivariate correlations and multiple regression analyses were performed to determine the organizational and nursing factors associated with MNC.

Population: Inpatient staff nurses currently working in Alabama were included in the study sample. A total of 950 completed nurse surveys were included for analysis.

Results: Of the 31 possible MNC activities, nurses missed, on average, approximately 17 care activities to some degree within their past seven working shifts. Activities of care requiring less clinical judgment, including timely response to patient and family requests and providing emotional and psychological support, were the most frequently reported MNC activities. Job satisfaction, unit type, nurse-reported quality of care, and the five domains of the nursing work environment were all significantly associated with MNC. Models with these variables resulted in $R^{2}$ values of 0.203 - 0.302 , indicating the model of MNC explains $20.3 \%$ to $30.2 \%$ of the variability of predictors around its mean.

Conclusions: MNC is a frequently occurring phenomenon in Alabama, threatening the quality of care hospitalized patients receive from nurses. Fortunately, the organizational and nursing factors associated with $\mathrm{MNC}$ are modifiable and can inform strategies to decrease the prevalence of MNC.
Implications for Clinical Practice and Regulation: Many of the nursing and organizational factors identified in this study that are associated with MNC are actionable. We recommend that nursing administration, nursing leaders, and policy makers consider regularly monitoring MNC as a quality measure and modifying elements of the nursing work environment to decrease the frequency of MNC.

\section{Machine Learning-Based 30-Day and 1-Year Mortality Prediction After Discharge From Inpatient Rehabilitation for Hip Fracture Patients}

Authors: Michael P. Cary, Jr., PhD; Farica Zhuang, MS; Rachel Lea Draelos, MS; Wei Pan, PhD; Sathya Amarasekara, MS; Brian J. Douthit, MSN, RN-BC; Yunah Kang; Cathleen S. Colón-Emeric, MD, MHS

Objective: To develop machine learning models designed to predict 30-day and 1-year mortality for Medicare beneficiaries aged 65 years or older treated in inpatient rehabilitation facilities (IRFs) following hospitalization for hip fracture.

Study Design: A retrospective design/cohort analysis of the Centers for Medicare and Medicaid Services Inpatient Rehabilitation Facility-Patient Assessment Instrument first-admission data were used for 17,140 persons admitted to Medicare-certified IRFs in 2015 following hospitalization for hip fracture. Independent variables included patient characteristics such as sociodemographic (i.e., age, gender, race, and social support) and clinical factors (i.e., functional status at admission, chronic conditions) as well as utilization (i.e., length of stay). The dependent variables were 30-day and 1-year mortality. We trained and evaluated two types of classification models_-logistic regression and a multilayer perceptron (MLP) — that used multivariable combinations of patient data described above to predict the probability of 30-day and 1-year mortality. Four measures were used to determine model performance: (a) accuracy (ACC), (b) area under the receiver operating characteristic (AUROC), (c) average precision (AVG PREC), and (d) calibration slope.

Population: The analytic sample included 17,140 hip fracture patients who received postacute care services in 1,112 IRFs.

Results: The best predictive model for 30-day mortality was $\operatorname{MLP}(\mathrm{ACC}=0.743, \mathrm{AUROC}=0.762, \mathrm{AVG}$ PREC $=0.103$, slope $=1.14)$ versus logistic regression $(A C C=0.78$, AUROC $=0.76$, AVG PREC $=0.097$, slope $=1.20$ ), and for 1-year mortality was logistic regression $(\mathrm{ACC}=0.684, \mathrm{AUROC}=0.756$, AVG PREC $=0.326$, slope $=0.957)$ versus $\mathrm{MLP}(\mathrm{ACC}=0.682$, AUROC $=0.757$, AVG PREC $=0.326$, slope $=0.962$ ). Both models showed fair predictive power and performed similarly across all four measures.

Conclusions: This study presented analysis of prognostic factors of hip fracture mortality using two different machine learning models. Although model evaluation suggested that MLP may yield slightly better (although not statistically significant) accuracy when compared to logistic regression, both models have high AUROCs 
and good calibration and can serve as valuable tools for accurately identifying patients with hip fracture at high risk for 30-day and 1-year mortality.

Implications for Clinical Practice and Regulation: Logistic regression and MLP models have similar predictive power and may be implemented to reduce cognitive burden. These models can be trained on local data to enhance clinical specificity in mortality prediction so that palliative care resources may be allocated more effectively.

\section{Analysis of ProviderType at the Time of Admission for Low- Risk Pregnant Patients and Birth Outcomes}

Author: Rachel Breman, PhD, MPH, RN

Objective: In the United States, approximately one third of births are cesarean. Furthermore, cesarean delivery is the most frequent surgical procedure. Reducing the rate of cesarean delivery in a generally healthy population of patients is a key focus of efforts to decrease cesarean birth nationwide. Previous research suggests that hospital admission in active labor among healthy women can reduce the use of medical interventions and cesarean birth. The purpose of this study was to examine the influence of hospital admission management and maternal sociodemographic factors on labor and birth outcomes among groups of women who were triaged for hospital admission by a rotating staff of midwife versus physician providers.

Study Design: Retrospective cohort study.

Population: Low-risk pregnant women with a term gestation fetus in a vertex position $(N=335)$ admitted to a community hospital for labor in 2015 by a nurse-midwife $(n=175)$ or physician provider $(n=160)$.

Results: Forty-three percent (74/172) of patients admitted by midwives and $93 \%(147 / 158)$ of patients admitted by physicians went on to deliver with the same type of provider. Patients admitted by midwives had more advanced cervical dilatation ( $4.1 \mathrm{~cm}$ vs. 3.3 $\mathrm{cm} ; p<.05)$ and effacement (88.2\% vs. $80.8 \% ; p<.01)$ at admission and were less likely to receive labor augmentation or epidural (39.8\% vs. $55.1 \%$ and $77.0 \%$ vs. $93.0 \%$, respectively) compared with patients admitted by physicians. In multivariate analysis controlling for influence of patient sociodemographic factors, women admitted by physicians were 3 times as likely to have a cesarean birth as those admitted by a midwife. Among patients who gave birth in the first 24 hours after triage, the median length of labor in those admitted by midwives was nearly 3 hours shorter (10.9 hours, IQR $=6.9-15.4)$ than those admitted by physicians $(13.5$ hours, IQR $=8.2-18.1$. In a subanalysis of patients having a vaginal birth, there was no difference in labor length by provider type, but public insurance compared to private insurance was associated with shorter labor duration (median, 9.45 hours for public insurance and 11.77 hours for private insurance) after accounting for triage provider type, sociodemographic, and labor factors.

Conclusions: Patients triaged by midwives were less likely to experience labor augmentation, epidural, and a cesarean birth compared

Volume 11/Issue 2 July 2020 to similar women triaged by physicians. Publicly insured women spent less time in the hospital in labor preceding vaginal birth.

Implications for Clinical Practice and Regulation: Use of midwives in labor triage units may potentially decrease cesarean rates and associated costs. Future studies are needed to explore reasons why publicly insured patients who have vaginal birth may spend less time laboring in the hospital and the possible cost implications.

\section{The Importance of Nursing Work Environments and Job Satisfaction in Reducing Preventable Nurse Turnover}

Authors: Tanekkia M. Taylor-Clark, MMAS, MSN, RN, CMSRN; Pauline A. Swiger, PhD, RN, CNL, CMSRN; Colleen V. Anusiewicz, BSN, RN; Caitlyn M. Campbell, BSN, RN; Lori A. Loan, PhD, RN, FAAN; Danielle M. Olds, PhD, MPH, RN, CIC; Sara T. Breckenridge-Sproat, PhD, RN; Dheeraj Raju, PhD, MS, MSIE; Patricia A. Patrician, PhD, RN, FAAN

Objective: Nurse turnover rates within the United States average $19.1 \%$ and can cost a hospital up to $\$ 7.86$ million annually. Other negative consequences of nurse turnover are well documented and include adverse patient outcomes, such as increased falls and mortality rates and decreased satisfaction with healthcare, and adverse nurse outcomes, including increased burnout and decreased job satisfaction. The inability to retain experienced, knowledgeable nursing personnel has detrimental effects on overall healthcare delivery system performance, not only in the civilian community but within the Military Healthcare System (MHS) as well. The purpose of this study was to conceptually define potentially preventable losses (PPL) and examine the associations between the nursing work environment, job satisfaction, unit characteristics (i.e., medical-surgical vs intensive care units), nursing roles (i.e., registered nurse $[\mathrm{RN}]$ and licensed practical nurse [LPN]), and PPL.

Study Design: This descriptive and correlational secondary data analysis uses Annual Nursing Workforce Survey data extracted for a multifocus, longitudinal, descriptive, and correlational parent study of the impact of nursing on patient outcomes. The sample for this analysis contains 290 observations from 2011 and 246 from 2014 from civilian and military RNs and LPNs employed in 23 U.S. Army hospitals. Descriptive statistics, correlations, and data mining predictive modeling techniques were used to evaluate the relationships between the outcome variable and the predictor variables.

Results: PPL further categorizes intent to leave reasons as a result of organizational structure, compensation, or working environment that could have potentially been prevented given timely identification of issues and appropriate intervention. More than $45 \%$ of respondents indicated that they intended to leave their U.S. Army hospitals in 2011 and 2014. Of those that intended to leave, PPL reasons accounted for $50 \%$ of all reasons in both years. Dissatisfaction with management, work environment, and personal reasons were the top three PPL reasons why respondents intended to leave, accounting for $74 \%$ of all PPL reasons in 2011 and 2014. 
In 2011, job satisfaction, Nurse Participation in Hospital Affairs, Nurse Foundations of Quality Care, Nurse Manager Leadership Support, Staff and Resource Adequacy, and Subscale Composite scores were significantly lower for respondents who indicated they were leaving for PPL reasons. Results were similar in 2014, with Nurse Physician Relations instead of Staff and Resource Adequacy scores being significantly lower. The random forests model indicated job satisfaction and Nurse Manager Leadership Support were the most important predictor variables for PPL in 2011 and 2014, respectively.

Implications for Clinical Practice and Regulation: These findings add to our understanding a conceptual definition of PPL and potentially preventable reasons why nurses in the MHS intend to leave. These findings may also assist in the development of actionable nurse retention strategies and intervention studies to ultimately reduce nurse turnover.

\section{Nurse Pain: A Practice and Policy Issue}

Authors: Jessica G. Rainbow, PhD, RN; Katherine M. Dudding, $\mathrm{PhD}$, RN; Claire Bethel, MSN, RN-BC

Objective: The aims of this study were to examine the prevalence, locations, and severity of nurse pain; explore the impact of nurse pain on performance at work; and describe nurse strategies for coping with physical and psychological pain. The overall prevalence of nurses working in pain is unknown because pain prevalence is site specific; however, back pain, a commonly cited source of pain, occurs in $35 \%$ to $80 \%$ of nurses.

Study Design: A cross-sectional survey of nurses recruited via social media was conducted in 2019. Survey items included participant demographics, locations and severity of pain, impact of pain on performance at work, and coping strategies. Descriptive analysis was completed.

Population: Our convenience sample consisted of direct-patient care nurses $(N=3,226)$ in the United States. Our sample was predominantly female, represented by all 50 states, and ranged in age from 20 to 76 years ( $M=41$ years). More than half the nurses surveyed $(n=1,602)$ had 1 to 10 years of nursing experience. The education level of the nurses varied, but the majority had a baccalaureate degree $(n=1,727 ; 53.7 \%)$. The majority worked in the hospital setting $(n=2,478 ; 76.9 \%)$.

Results: The majority ( $n=2,843 ; 88 \%$ ) of survey participants responded that they were currently experiencing or had experienced pain in the past week. The most frequently reported locations of their pain were back, neck, shoulders, knees, and feet. The average pain level was four out of 10 . Approximately $85 \%$ of nurses reported more than one location of pain. Participants reported managing their pain by over-the-counter medications $(n=2,140$; 77.7\%), nonpharmacological pain management ( $n=1,413 ; 51.3 \%)$, and prescription medications $(n=694 ; 25.2 \%)$. Additionally, over one third of nurses $(n=996 ; 35 \%)$ reported using prescription drugs, marijuana, or alcohol as a strategy to cope with their pain. On average, $45 \%$ of nurses stated their pain impacted their work performance and $25 \%$ stated their pain impacted direct-patient care.

Conclusions: The prevalence, severity, and impact of nurse pain on performance at work highlights the importance of addressing and investigating nurse pain. Workplace interventions to address nurse pain have mostly focused on back pain through lifting devices; however, nurses continue to have high levels of back pain and pain in other locations. The propensity of unhealthy coping strategies by nurses is concerning and warrants further investigation.

Implications for Clinical Practice and Regulation: In the wake of the opioid epidemic, future interventions should investigate additional pain sites and encourage healthy coping strategies for nurse pain. The legalization of marijuana in many states also presents a potential new area of research and policy as many practice environments are considering how to handle healthcare provider marijuana use. State boards of nursing have long sought to minimize and address substance use disorders given nurses' access to controlled substances. Unrelieved pain among nurses combined with their work demands, as well as access and knowledge of pharmaceuticals, may contribute to the higher suicide rate of nurses by gender than their U.S. population counterparts, which is more often carried out by pharmaceutical overdose. As the nursing workforce ages, interventions to reduce nurse pain are key to sustaining the workforce.

\section{Flexible Nurse Staffing on Hospital Units:The Cost and Consequences of Different Strategies-A Simulation Modeling Study}

Authors: Peter Griffiths, PhD, RN, and Safer Nursing Care Tool Study Group: Jane Ball, PhD; Rosemary Chable, MSc, RN; Andrew Dimech, MSc, RN; Yvonne Jeffrey; RN; Jeremy Jones, $\mathrm{PhD}$; Natalie Pattison, PhD, RN; Alejandra Recio Saucedo, PhD; Christina Saville, PhD; Nicola Sinden, MSc, RN; Thomas Monks, $\mathrm{PhD}$

Objective: The objective of this study was to model the consequences and costs of using a recommended staffing tool (the Safer Nursing Care Tool) to guide hiring, scheduling, and deployment decisions in English acute hospitals, specifically exploring how different baseline staffing levels affect costs, the likelihood of critical staffing shortfalls, and mortality risk.

Study Design: We compared flexible baseline staff schedules (set to meet $80 \%$ of the mean demand) with staffing schedules set to meet mean demand (the standard approach) and schedules set to match peaks in demand (90th percentile). In all cases, floating from units with low demand to units with high demand and temporary hires were used to provide a flexible response when demand exceeded scheduled supply on any unit on any shift. Data from a multicenter observational study of staffing and patient acuity/ dependency (26,362 unit $\times$ days of observations over 1 year) to provide parameters, including probability distributions for varying demands for nursing care, were used. A computer simulation was developed to model the effects of different baseline schedules and approaches to floating and use of temporary hires and over- 
time. The model was used to simulate daily staffing costs and the occurrence of critical ( $>15 \%)$ understaffing. It included realistic assumptions about the possibility of scheduled staff failing to show for work at short notice due to sickness and for constraints on the availability of temporary hires and overtime. The effects of any consequent short staffing, in terms of mortality risk and length of stay, were estimated using parameters from a recent longitudinal observational study in one of the participating hospitals. An economic model estimated the cost per life saved.

Population: General medical surgical wards in four public hospitals in England.

Results: In simulation experiments, "flexible (low)" schedules led to high rates of understaffing and adverse outcomes, even when temporary staff were readily available. "High" staffing baseline schedules were associated with reductions in understaffing and improved outcomes but higher costs. Under most assumptions, the cost per life saved from moving from a lower to a higher baseline schedule was below $£ 30,000$ (approximately $\$ 40,000$ USD). If unlimited availability of temporary staff is assumed, the harm associated with low staff schedules was minimized, but net cost per life saved for higher staffing levels was also low: $£ 946$ ( $\$ 1,250$ USD) for standard vs low and $£ 5524$ (\$7,300 USD) for high versus standard.

Conclusions: Cost savings from a policy of flexible staffing with low baseline schedules are largely achieved by below-adequate staffing. Cost savings are eroded with the high temporary staff availability required to make such policies function safely. Higher baseline schedules are cost-effective.

Implications for Clinical Practice and Regulation: In the face of nursing shortages, which are endemic in England and many other jurisdictions, these findings illustrate the possible consequences of short staffing and illustrate that higher nursing staff levels remain a desirable goal even if staff redeployment and use of temporary staff can be used to fill gaps in rosters. The common basis of staffing tools, where mean averages are used to guide staffing decisions, is questioned.

Funding Source and Disclaimer: This abstract presents independent research funded by the United Kingdom's National Institute for Health Research (NIHR) Health Services and Delivery Research Programme (award number 14/194/21). The views and opinions expressed are those of the authors and do not necessarily reflect those of the National Health Service, the NIHR, the NIHR Evaluation, Trials and Studies Coordinating Centre, or the Department of Health and Social Care.

\section{Associations Between Cross-sector Collaboration and Family Outcomes in Evidence-Based Nurse Home-Visiting}

Authors: Venice Ng Williams, PhD, MPH; Beth M. McManus, PT, MPH, ScD; Ashley Brooks-Russell, PhD, MPH; Elly Yost, RN, MSN, PNP; David L. Olds, PhD; Gregory J. Tung, PhD, $\mathrm{MPH}$

Volume 11/Issue 2 July 2020
Objective: Home-visiting prevention programs must coordinate with other community providers to be most effective. However, the associations between collaboration and program outcomes are not well understood. Nurse-Family Partnership (NFP) is an evidence-based prenatal and early infancy home-visitation program delivered by nurses to low-income, first-time mothers. NFP is implemented by more than 250 agencies across the United States by public health departments, community-based organizations, and healthcare systems. This study aimed to examine the associations between cross-sector collaboration NFP and client-level outcomes.

Study Design: We used 2018 survey data with NFP supervisors that assessed agency-level collaboration, as measured by relational coordination and structural integration with nine community provider types (including obstetrics care, substance use treatment, and child welfare). We paired the collaboration survey data with NFP program implementation data from 2014 to $2019(N=36,900)$ to examine outcomes including client retention, client smoking cessation, and childhood injury. We used random-intercept models with nurse-level random effects, controlling for client-level demographics and health, nurse-level demographics, and agency-level administrative and geographic factors.

Population: Sample NFP clients were on average 23 years old and predominately single. Of sample clients, $47.5 \%$ were White, 33\% were African American, 31\% identified as Hispanic, $11.5 \%$ reported another race, and $8 \%$ declined reporting.

Results: Consistent with past research, we found unmarried women, African-American women, and those visited by nurses who ceased employment with NFP prior to the client's child's birth were more likely to drop out of the NFP program, whereas older women and high school graduates were more likely to remain in NFP. Greater relational coordination with substance use treatment providers $(O R=1.168 ; p<.001)$ and stronger structural integration with child protective services (CPS) $(O R=1.065$, $p<.001)$ were associated with improved client retention at birth, even after adjusting for multilevel factors. Structural integration with CPS remains significantly associated with client retention at 12-months postpartum. Stronger nurse coordination with the Special Supplemental Nutrition Program for Women, Infants, and Children (WIC) $(O R=1.097, p<.05)$ as well as with substance use treatment providers $(O R=1.108, \mathrm{p}<.05)$ were associated with increased self-reported prenatal smoking cessation. Stronger nurse coordination with WIC $(O R=0.863, p<.01)$ and greater integration with mental health providers $(O R=0.975, \mathrm{p}<.05)$ were associated with decreased probability of self-reported emergency department use for childhood injury.

Conclusions: Improving healthcare through relationships with other care providers is necessary to enhance the experience and outcomes of patients, particularly among high-need complex populations. This study provides early results suggesting cross-sector collaboration in a home-visiting setting that bridges healthcare and addresses social determinants of health has potential to improve 
the retention of clients. More research is needed to understand how collaboration may improve maternal-reported behaviors like smoking cessation and emergency department use for childhood injury. Implications for Clinical Practice and Regulation: Our findings inform efforts to increase efficient delivery of prevention programs like NFP through intentional collaboration with cross-sectors, as well as for future agency development for NFP expansion, especially as the program moves toward integration with healthcare delivery systems.

\section{Alleviation of Primary Care Provider Burnout Through Co-Management Care Delivery Models}

Authors: Allison Norful, PhD, RN, ANP-BC; Yun He, BM; Adam Rosenfeld, BA; Cilgy Abraham, BS, RN

Objective: Primary care providers (PCPs) are increasingly reporting burnout when trying to meet demands for patient care. Previous evidence suggests that individual PCPs require 21 hours per day to complete all care responsibilities. Policymakers are calling for novel delivery models to help meet the care demand. One emerging model, provider co-management, has been shown to yield optimal clinical outcomes. Co-management is structured with two PCPs, often interdisciplinary dyads (e.g., physicians and nurse practitioners), who share care responsibilities for the same patient. Theoretically, co-management is comprised of 3 dimensions: effective communication, mutual respect/trust, and shared philosophy of care. It remains unclear, however, whether co-management alleviates PCP burnout. Thus, the purpose of this study was to determine the impact of co-management on PCP burnout and job satisfaction.

Study Design: We conducted a cross-sectional survey of PCPs in New York State using Dillman methodology. Paper surveys were mailed to a LISTSERV of randomly selected PCPs obtained from IQVIA, the largest provider reference database in the United States. Postcard reminders were sent after 3 weeks and then a second survey was sent to nonrespondents. The Provider Co-Management Index (PCMI) $(r=.81)$ was used to measure comanagement with higher scores, indicating more effective co-management. The Maslach Burnout Inventory $(r=.90)$ was used to scale self-reported burnout. We calculated descriptive statistics and crude odds ratios from bivariate logistic regression models. Next, adjusted odds ratios were calculated from multivariable logistic models controlling for provider and practice characteristics.

Population: Our sample included 333 PCPs across New York State, made up of physicians ( $n=95)$, nurse practitioners $(n=157)$, and physician assistants $(n=81)$. Half of participants worked in provider-owned practices $(50 \%)$ and had more than 10 years of experience $(56 \%)$.

Results: Almost $30 \%$ of PCPs reported job dissatisfaction and burnout. There were no significant differences in responses between workforce types. PCPs who reported burnout had $71 \%$ less odds of job satisfaction $(O R=0.29,95 \% \mathrm{CI}=0.17,0.49)$. Similarly, participants who reported burnout had more than three times the odds of planning to leave their position in the next year compared to those who did not report burnout $(O R=3.19$, 95\% $\mathrm{CI}=1.75,5.83)$. With each unit increase in total PCMI score, there was 1.05 times the odds of job satisfaction $(O R=1.05,95 \%$ $\mathrm{CI}=1.02,1.07)$. The magnitude of effect became larger when looking at PCMI subscales representing each co-management dimension. Each unit increase in one PCMI subscale was associated with $15 \%$ less odds of burnout and $10 \%$ less odds of intention to leave a position within 1 year. Estimates in adjusted models holding controls constant remained similar to those in the unadjusted models.

Conclusions: The more effective that co-management is between PCPs, the less significantly associated are provider burnout, job dissatisfaction, and intent to leave current position. Provider comanagement may be a promising approach to help alleviate PCP burnout.

Implications for Clinical Practice and Regulation: Attention to interdisciplinary team compositions and policies that enable comanagement care delivery dimensions are recommended to help alleviate burnout. Further research, including comparative and cost-effectiveness studies, are warranted to determine financial sustainability of organizations that implement co-management models.

\section{Development and Validation of Competencies for Registered Nurses in Canada: A Delphi Study}

Authors: Julia Lukewich, RN, PhD; Michelle Allard, RN; Lisa Ashley, RN MEd; Kris Aubrey-Bassler, PhD; Denise BryantLukosius, RN, PhD; Treena Klassen, RN, MEd; Tanya Magee, RN, BN; Ruth Martin-Misener, NP, FAAN, PhD; Maria Mathews, PhD; Allison Norful, RN, ANP-BC, PhD; Marie-Eve Poitras, RN, PhD; Josette Roussel, RN, MSc, MEd; Dana Ryan, MA; Ruth Schofield, RN, MSc(T); Joan Tranmer, RN, PhD; Ruta Valaitis, RN, PhD; Sabrina T. Wong, RN, PhD

Objective: The increasing volume of registered nurses (RNs) employed in primary care settings may offer solutions to overcoming care delivery complexities facing international healthcare systems, including Canada. There is growing evidence that RNs in primary care improve access, reduce costs, and promote higher quality care; however, there is a lack of clarity surrounding the effective deployment of the $\mathrm{RN}$ role, specifically in primary care settings. The purpose of this study was to develop and validate a set of national primary care RN competencies in Canada. The competencies aim to better support the integration and optimization of the role of RNs in primary care.

Study Design: This study employed a Delphi survey process. An initial draft of competencies consisting of 49 statements was created and guided by international literature, a panel of key informants (i.e., researchers, stakeholders, project partners), and competencies of related practice areas, such as public health nursing and home care. Using an online survey platform, participants rated the importance of each competency statement using a 
6-point Likert scale ( 1 = not important; 6 = extremely important) and offered written feedback/suggestions. Statements that did not reach consensus ( $\geq 80 \%$ agreement or mean $\geq 5.0$ ) were modified and sent to participants for a second (final) Delphi round.

Population: Canadian nurses with knowledge and expertise in primary care were identified through snowball sampling, online searches, and professional organizations (e.g., Canadian Family Practice Nurses Association) and invited to participate in the survey through email correspondence $(n=137)$. Individuals represented all domains of nursing (i.e., clinical, research, education, policy, administrators) and all Canadian provinces and territories. The survey was available in both English and French.

Results: The first survey was completed by 63\% (86/137) of invited participants (April and May 2019), and 84\% (72/86) of these firstround participants completed the second follow-up survey (June through August 2019). Most competencies $(n=45)$ achieved agreement after the first survey $(M=5.43, S D=0.191)$; one statement was dropped and two were combined following the second round. The finalized competency list consisted of 47 distinct statements organized across 6 domains (Professionalism; Clinical Practice; Communication; Collaboration and Partnership; Quality Assurance, Evaluation, and Research; Leadership).

Conclusions: A concise and preliminarily validated set of primary care RN competencies was developed. These competencies may be used to guide nursing curricula, increase understanding of the primary care $\mathrm{RN}$ role, improve interprofessional team functioning, and are foundational to strengthening the $\mathrm{RN}$ workforce in primary care.

Implications for Clinical Practice and Regulation: Primary care $\mathrm{RN}$ competencies have tremendous value to relevant stakeholders, including international policymakers, to use as a framework to inform administrators, clinicians, and researchers for targeted integration and optimization of RNs within primary care. In addition, the competencies can help inform local, provincial, and national policies, including funding models. Currently, the Canadian Nurses Association plans to incorporate these competencies into the Community Health Nursing Certification Examination. Next steps involve conducting two cross-sectional surveys with academic deans/directors and front-line primary care RNs to assess the integration of competencies within Canadian nursing curricula and the performance/learning needs of RNs in primary care relative to these competencies. Future research will validate such competencies in other countries.

\section{Development of Lifestyle Modification Interventions to Reduce Hypertension in the African American Faith-Based Community Using a Community-Based Participatory Research Framework}

Authors: Linda Y. Kim, PhD, MSN, RN, PHN; Harriet Aronow, PhD; Mana Manoukian, MSN, RN, AGCNS-BC; Brennan Spiegel, MD, MSHS; Bernice Coleman, PhD, ACNP-BC, FAHA, FAAN

Volume 11/Issue 2 July 2020
Objective: When planning hypertension interventions for African Americans with the goal of modifying behavior, one must be particularly sensitive to building trust and to the preferences and choices of the clients. Community-based participatory research (CBPR) builds a foundation of trust between investigators and participants by eliciting contributions from community members into the research process. The purpose of this study was to apply the CBPR framework to engage the African American faith-based community in the development of a health education program to address hypertension using both group learning and activities based in virtual reality (VR).

Study Design: Applying a CBPR framework, an expert panel consisting of members of a faith-based community along with the Council of Black Nurses (Los Angeles Chapter) was assembled and co-led by the community pastor and the research team from a medical center that serves this community. Two modified Delphi exercises elicited input regarding food preferences for the VR, and a 90-minute focus group gathered input on health, exercise, and stress management. The focus group transcript was analyzed using content analysis methodology and the results were used to develop the course content.

Population: Fifty-eight participants aged 18 to 85 years were recruited from a predominately African American church in Los Angeles, California, where all research activities were held.

Results: With participant input, the VR intervention included education on adverse impacts of high sodium diets on blood pressure (BP) and body organs, culturally common low-sodium food alternatives, and stress management meditation and mindfulness exercises (recorded by the pastor). Group preference identified content for a 12-session course that included (a) diet and sodium intake, (b) BP monitoring, (c) healthy lifestyle, (d) exercise, (e) diabetes, (f) sleep and stress management, ( $g$ ) genetics and health, (h) a cooking class, and (i) tai chi. Initial interest and participation were so high that study recruitment targets were expanded. Participant feedback was collected during classes. Feedback on the course was positive. Despite having technical support, participants found the VR component to be challenging; thus, VR use was suboptimal.

Conclusions: Although there were several challenges associated with implementation of the VR component of the intervention with this cohort, lessons learned provide insight on how to incorporate community input into the development of technologies meant to support hypertension control in vulnerable populations. The CBPR approach was used to successfully partner with a faithbased community to develop interventions aimed at health promotion and disease prevention.

Implications for Clinical Practice and Regulation: Churches are excellent venues for delivery of community-based lifestyle interventions because of their pre-existing social networks, consistent attendance, and a faith-based focus in enhancing the connection between mind, body, and spirit. Ideally, the impetus for changing behavior should come from within the targeted community 
itself, and the intervention should be built around the preferences and choices of that community. CBPR involving community stakeholders provides the foundation for collaborations that are responsive to the specific needs of a community and can improve understanding of the underlying contributors of persistent health disparities.

\section{A Community Voice in Response to the Opioid Crisis Impacting Children in Rural America}

Authors: Jane Bolin, Jodie Gary, Cynthia Weston, Nancy Downing, Allison Pittman, Cherrie Pullium

Objective: The goal of this mixed methods study was to conduct both quantitative and qualitative research with dissemination to community partners toward forming a united regional consortium focused on increasing access to opioid use disorder (OUD) prevention, treatment, and recovery, ultimately improving the health and wellbeing of children and families. An overarching goal is to address challenges of delivering care in underserved and rural communities.

Design: We employed mixed methods to examine longitudinal trends of opioid-related admissions in the rural Gulf Bend region over a 6-year period. We then conducted several weeks' worth of focus groups and structured interviews in each of the counties. We utilized a semi-structured interview guide to engage the community in identifying the scope of OUD and its impact on children, including neonates. Focus groups explored gaps in available treatment and recovery services and in critical resources and workforce. The project was designated non-human subjects research by the Texas A\&M Institutional Review Board.

Population/Sample: Our study was conducted in seven counties in the rural Gulf Bend region of Texas that are impacted by rising rates of opioid misuse and exacerbated by co-occurring use of other narcotic, controlled substances, as well as alcohol. Children and their safety were a focus of our study.

Findings: Fifteen focus group interviews with over 100 stakeholders included physicians, nurses, hospital administrators, advocacy groups, treatment centers, child protective services, first responders, law enforcement, social workers, faith-based groups, and elected officials. The outcome was an OUD community assessment of existing prevention, treatment, and recovery resources and access to resources; assets and opportunities; and gaps and constraints. Strategies identified included targeted OUD education and training, access to medication-assisted treatment and peer-topeer recovery support, and access to mental health resources. Use of innovative telehealth programs are planned to address gaps in services.

Conclusion: OUD is a significant health and safety issue for children and their families. Neonates are born addicted and families are impacted, as are hospitals, schools, and law enforcement. Implications include collaborative partnership development and gathering a community voice to address gaps in prevention, treatment, recovery, and workforce. Community-driven solutions were identified to build capacity through existing community strengths and shared knowledge and communication of resources. Innovative strategies include the use of peer-to-peer parent recovery support and nurse-led telehealth programs.

Implications: Our research demonstrated that community stakeholders believe that OUD is contributing to child neglect and abuse and contributes to economic insecurity, which severely impacts children. Infants born to mothers with OUD are at risk for neonatal abstinence syndrome. Parental OUD can also lead to separation from children due to incarceration, treatment, hospitalization, or child removal. Child removal is traumatic, and foster care placement may contribute to adverse mental and physical health outcomes for children. Adverse childhood experiences related to parental substance abuse or separation are associated with developmental disruption, risk behaviors, adverse physical and mental health conditions, and increased healthcare utilization.

Funding: Health Resources and Services Administration Rural Community Opioid Response Planning Grant; awarded June 2019 to Jane Bolin, primary investigator.

\section{Exploring the Potential of Rehabilitating Nurses and Nurse Practitioners Prosecuted for Fraud and Abuse}

Authors: Jane Bolin, Nancy Fahrenwald, Cindy Weston, and Debra Matthews

Objective: This study addresses two national problems. First, in the United States, especially in Texas, healthcare suffers from a severe shortage of nurses. Second, there are more than 28,600 nurses, including advanced practice nurses, nationally who have run afoul of the law and thereby lost their license and privilege to practice their professions. The central goals and aims of this research were to examine the OIG's Fraud and Abuse list of sanctioned healthcare providers (publicly available) and to analyze the characteristics of nurses who have lost their license or have been barred from participating in Medicare and Medicaid.

Design: We employed mixed methods to explore what interventions may be effective for rehabilitating sanctioned nurses and learn from these individuals what factors influenced them to choose crime or deviant behavior after investing so much time, energy, and financial resources in their professional training. In future studies, we will explore what measures, requirements, and restraints should be put in place for rehabilitation of those who were once licensed healthcare providers in good standing, including surveying state licensing boards.

Population/Sample: Nurses, including nurse practitioners and doctors of nursing practice, who have been convicted of behavior leading to their exclusion from practice in the United States. As of January 2020, more than 28,600 nurses are listed in the Office of the Inspector General's (OIG's) list of sanctioned and excluded providers.

Findings: Currently, more than 28,000 nurses have been convicted or sanctioned for prohibited conduct leading to their exclusion from practicing in programs that are eligible for Medicare 
and Medicaid payment. Twenty-seven NPs have been excluded/ convicted, 20 nursing firms, and 28,639 individuals designed as nurses. California and Texas lead the nation in excluded nurses at 2,571 and 2,402, respectively. Tragically, 3,847 nurses have been convicted of patient abuse, 249 have been convicted of healthcare fraud, and 20,111 have had their licenses revoked by their state boards.

Conclusion: Shortages of nurses are especially severe in rural and underserved parts of Texas and the United States. Rural citizens are often 1 to 2 hours from the closest healthcare provider. As a profession, we need to explore whether it is possible to address the nation's severe health care provider shortage by redeeming nurses who lost their license through financial fraud or other unlawful behavior. Programs for rehabilitating nurses and subject to appropriate oversight and restriction may address the current severe provider shortage in medically underserved areas.

Implications: The potential of such a program is enormous and paradigm changing. However, we must first identify those who can be successfully rehabilitated. Researchers in this multi-phase study will next explore what interventions may be effective for rehabilitating sanctioned providers to determine what factors influenced them to choose crime or deviant behavior. It is imperative that we explore what measures should be put in place for rehabilitation of those who were once licensed in good standing. We will also survey experts from state licensing boards to determine what approaches might be employed to rehabilitate even a fraction of the more than 28,600 + sanctioned nurses sitting idle on the sidelines.

Funding: The President's Excellence Fund, Triad-3 Initiative, 2020, provided support for this research.

\section{Maldistribution of the Population Health Nursing Workforce in North Carolina: Implications for the Medicaid Program's Plan to Roll Out Healthy Pilots to Address the Social Determinants of Health}

Author: Jacqueline Nikpour

Objective: In a healthcare system rapidly shifting from rewarding volume to incentivizing value, policymakers, payers, and health systems are increasingly focused on addressing patients "upstream" social determinants of health (SDOH). Typically, Medicaid funds cannot be used for nonmedical interventions, but North Carolina has received permission from the Centers for Medicare and Medicaid Services, through an 1115 waiver, to use Medicaid funds for "Healthy Opportunity Pilots" that address the SDOH. Due to nurses' education, broad scope of practice, and focus on holistic, whole-person care, the workforce is uniquely positioned to address housing instability, food insecurity, and other population health issues. Yet little evidence exists describing the supply, distribution, education, and practice characteristics of the nursing workforce available to address these challenges. This information is critical to identify where nursing workforce gaps exist that may jeopardize an effective rollout of North Carolina's Healthy Opportunity Pilots.
Design: This study used 2018 North Carolina licensure data to describe the demographic, education, and geographic characteristics of the nursing workforce in the following SDOH settings: ambulatory care, public/community health, occupational health, long-term care, home health, correctional facilities, and schoolbased health. We compared these "population health" nurses to those employed in hospital settings using chi-square and $t$ tests. North Carolina's 100 counties and 6 Medicaid regions were used as the units of analyses for measures of supply and population health need. We categorized Medicaid regions as high income, moderate income, and low income based on their 20th percentile income level and performed one-way ANOVA tests to determine differences in nurse supply. We then merged licensure data, at the county level, with the 2018 County Health Rankings to compare the distribution of population health nurses with population health needs using Spearman correlation analyses.

Population/Sample: 93,571 RNs in active practice in North Carolina in 2018.

Findings: In 2018, nearly 30\% of nurses were practicing in a population health setting, compared with $54 \%$ employed in hospitals. Compared to hospital nurses, population health nurses were more likely to be female ( $94.7 \%$ vs. $89.5 \%, p<.001)$ and less likely to have a bachelor's degree or higher $(35.5 \%$ vs. $65.5 \%, p<.001)$. There was no difference in nurse race between hospitals and population health settings $(p=.59)$. One-way ANOVA tests revealed that population health nurses were significantly more likely to work in high-income regions when compared with moderateincome and low-income regions $(F=4.47, p=.01)$, potentially due to the fact that $49.6 \%$ of the state population is located in these two counties. At the county level, a lower supply of population health nurses was associated with higher rates of uninsured people $(p=.02)$, unemployment $(p<.0001)$, child poverty $(p<.0001)$, low income $(p<.0001)$, poor or fair health $(p=.004)$, physically unhealthy days $(p=.0006)$, mentally unhealthy days $(p=.0009)$, air pollution $(p<.0001)$, and preventable hospitalizations $(p=.0009)$.

Conclusion: North Carolina's population health nurse workforce is not well distributed relative to the state's population with higher SDOH needs.

Implications: As North Carolina prepares to implement its 1115 health pilots, workforce planning initiatives are needed to more equitably distribute the supply of population health nurses across the state.

\section{An Interagency, Interprofessional Project to Understand Food Insecurity Among Older Adults}

Authors: Shirley Girouard, PhD, RN, FAAN; Michele Solloway, PhD, MPA

Objective: A state government and an urban university collaborative project seeks to understand and promote knowledge about food insecurity (FI) among older adults. We sought to develop tools to assess and increase FI screening and referrals as well as 
increase access to nutrition education and food resources with the ultimate goal of reducing FI-related health disparities.

Population/Sample: Adults aged 65 or older in a large, diverse urban community with high health disparities.

Findings: A total of 390 surveys were collected from participants at 12 health fairs and 2 community presentations. The mean age of respondents was 74.5 years. The majority were women $(83 \%)$. More than half were Black (53\%), 24\% were Caribbean, and 10\% were mixed or "other." Approximately $23 \%$ were at high FI risk and $31 \%$ at moderate FI risk. Approximately $40 \%$ of respondents received food stamps. Among respondents with health conditions ( $n=220)$, more than half had high blood pressure $(56 \%)$ or high cholesterol (55\%); 43\%, diabetes; $27 \%$, heart disease; and $8 \%$, stroke. Three-quarters $(76 \%)$ had 3 or more conditions; $62 \%, 4$ or more; and $47 \%, 5$ or more. Notably, $15 \%$ of respondents had all 6 conditions listed. Almost 1 in 4 respondents indicated that obtaining healthy and sufficient food was hampered by health conditions $(24 \%)$ or mobility (22\%).

Conclusion: FI was documented as a significant problem and compounded in complexity for individuals with multiple medical conditions. FI is also interwoven with culture and literacy. Discerning levels of FI risk for triage and policy purposes appears viable. Phase I of the project was well received by all key stakeholders; therefore, Phase II was initiated. Additional support is sought to continue Phase I activities and develop a primary care assessment, referral, and follow-up model.

Implications: Developing a screening tool to differentiate levels of FI risk is necessary to develop tailored community-based interventions for specific communities. Data generated by the survey facilitates health professions student education as well as ongoing education for providers. Cultural, literacy, mobility, access, and health disparity issues associated with FI risk become addressed more consistently as a result. Identifying high FI patients in practice can also lead to improved triage and referral by differentiating patients who require more intensive individual counseling from those who could benefit from community resources and classes. Such policies, programs, and practices can improve healthy eating and thus improve health outcomes for older adults.

\section{Associations Among the Nursing Work Environment, Nurse- Reported Workplace Bullying, and Patient Outcomes}

Author: Colleen Anusiewicz

Objective: Nurses are instrumental in preventing adverse events through the delivery of high quality, safe patient care in an efficient and effective healthcare system. However, workplace bullying may undermine safety culture in the workplace, subsequently affecting nursing care and patient outcomes. The objective of this study was to explore the association between the nursing work environment and nurse-reported workplace bullying and the association between nurse-reported workplace bullying and patient outcomes, including nurse-reported quality of care and nursereported patient safety grade.
Design: We conducted a cross-sectional analysis using data obtained from the Alabama Hospital Staff Nurse Study. The nursing work environment was measured using the original Practice Environment Scale of the Nursing Work Index (PES-NWI) with five domains represented as subscales and a composite score. Nurse-reported workplace bullying status was identified as "yes" or "no" using the Short Negative Acts Questionnaire (SNAQ) through a latent class analysis. Nurse-reported quality of care and patient safety grade were measured using single-item measures. The responses were dichotomized into either "excellent/good" or "fair/poor" for quality of care, and either "favorable" or "unfavorable" for patient safety grade. Random effects logistic regressions were used to determine associations controlling for individual and employment characteristics. Odds ratios (ORs) and $95 \%$ confidence intervals (CIs) were obtained to examine the strength of associations. All statistical analyses were conducted using $\mathrm{R}$ version 3.4.3. Population/Sample: Inpatient staff nurses working throughout Alabama $(N=943)$.

Findings: Nurses in this study were predominately white (82.0\%) and female (89.6\%). The median age was 37 years. Most nurses held a bachelor's degree $(56.1 \%)$, had primarily worked as a nurse for a median of 8 years, and were full-time (84\%), permanent (97.7\%) employees working 12-hour (88.1\%) day shifts $(60.7 \%)$ with minimal overtime (2 hours per week). A total of 377 (40\%) nurses reported experiencing workplace bullying. Nurses primarily reported excellent/good quality of care $(84.1 \%)$ and a favorable patient safety grade $(69.5 \%)$. After controlling for individual and employment characteristics, a higher PES-NWI composite score was significantly associated with a lower risk of nurse-reported workplace bullying $(O R=0.16,95 \% \mathrm{CI}=0.12,0.22, p<.0001)$. Nurses experiencing workplace bullying were less likely to report good/excellent quality of care $(O R=0.28,95 \% \mathrm{CI}=0.18,0.44$, $p<.0001)$ or a favorable patient safety grade $(O R=0.36,95 \%$ $\mathrm{CI}=0.25,0.51, p<.0001)$. However, these patient outcome associations were mediated by the PES-NWI composite score $(O R=0.78,95 \% \mathrm{CI}=0.44,1.37$, and $O R=0.89,95 \% \mathrm{CI}=0.57$, $1.41, p=.6300$, respectively).

Conclusion: These findings suggest that improving the nursing work environment can potentially decrease nurse-reported workplace bullying and perhaps subsequently improve patient outcomes.

Implications: The nursing work environment consists of modifiable, organizational factors that either support or detract from a nurse's ability to provide safe, high-quality patient care. Further exploring specific aspects of nursing work environments using the PES-NWI can inform the development of targeted organizationallevel anti-bullying interventions. Such interventions, however, must include the improvement of the nursing work environment. 


\section{References}

Alvidrez, J., Castille, D., Laude-Sharp, M., Rosario, A., \& Tabor, D. (2019). The National Institute on Minority Health and Health Disparities Research Framework. American Journal of Public Health, 109(S1), S16-S20. https://doi.org/10.2105/AJPH.2018.304883

Braveman, P. (2006). Health disparities and health equity: Concepts and measurement. Annual Review of Public Health, 27(1), 167-194. https://doi.org/10.1146/annurev.publhealth.27.021405.102103

Carey, G., \& Crammond, B. (2015). Systems change for the social determinants of health. BMC Public Health, 15(662). https://doi. org/10.1186/s12889-015-1979-8.

World Health Organization. (n.d.). Year of the nurse and midwife 2020. https://www.who.int/campaigns/year-of-the-nurse-and-the-midwife-2020

Deonni Stolldorf, PhD, RN, is an Assistant Professor, School of Nursing, Vanderbilt University, Nasvhille, Tennessee, and Vice Chair, The Interdisciplinary Research Group on Nursing Issues (IRGNI). Hayley D. Germack, PhD, MHS, RN, is an Assistant Professor, School of Nursing, University of Pittsburgh (Pennsylvania). Jordan Harrison, PhD, RN, i sa Associate Policy Researcher, RAND Corporation, Pittsburgh, Pennsylvania. Kathryn Riman, BSN, RN, is a Doctoral Student, Center for Health Outcomes \& Policy Research, School of Nursing, University of Pennsylvania, Philadelphia. Heather Brom, PhD, RN, is a Postdoctoral Fellow, Center for Health Outcomes \& Policy Research, School of Nursing, University of Pennsylvania. Michael Cary, PhD, RN, is an Assistant Professor, School of Nursing, Duke University, Durham, North Carolina. Heather Gilmartin, PhD, RN, is a Nurse Scientist, Rocky Mountain Regional Veterans Affairs Medical Center, Aurora, Colorado, and a Clinical Assistant Professor, Colorado School of Public Health, University of Colorado - Anschutz. Tammie Jones, MS, FACHE, CMPE, is a Doctoral Student, George Mason University, Fairfax, Virginia. Allison Norful, PhD, RN, ANP-BC, is an Assistant Professor, School of Nursing, Columbia University, New York, New York. Allison Squires, PhD, RN, FAAN, is an Associate Professor, Rory Meyers College of Nursing, New York University; 2019-2020 Distinguished Nurse Scholar in Residence, National Academy of Medicine; and Chair, IRGNI. 